\title{
Review of: "A trusted approach for prediction of data link failure and intrusion detection in wireless sensor networks"
}

\author{
dalal hammod ${ }^{1}$ \\ 1 Universiti Malaysia Perlis \\ Potential competing interests: The author(s) declared that no potential competing interests exist.
}

accept 